\title{
Gestión y marketing del capital intelectual Estrategia para posicionar los postgrados en el siglo XXI
}

\author{
Ferrer y Arroyo, Mercedes*
}

\section{Resumen}

El contexto global-digital, de redes y virtualidad real y, la crisis sin precedentes que atraviesa el país, demandan respuestas innovadoras de la universidad y, la redefinición y flexibilización del postgrado, donde la gestión y el marketing del capital intelectual asume un rol estratégico. A partir del reconocimiento de esta situación, este articulo tiene por objeto responder cuatro preguntas: ¿Qué caracteriza al mundo global -relacional de hoy y que impacto y demandas de cambio genera en la universidad y en los postgrados? ¿Qué es y cómo gestiona la universidad su capital intelectual (Cl) para potenciar las capacidades innovadoras? ¿Cómo se gestiona el conocimiento en un mundo complejo? ¿Cómo pasar de la universidad del compromiso social a la universidad con rentabilidad social? Las respuestas se formulan a partir del análisis comparado de dos escenarios u opciones de gestión de la universidad y de los postgrados, de lo que son y de lo que deben ser, el escenario funcional o tendencial de la universidad de la excelencia con compromiso socialy, el escenario emergente del postgrado en red $y$, homologado internacionalmente, que gestiona y mercadea el capital intelectual de la universidad con rentabilidad social del tercer milenio y, se identifican los modelos de gestión implícitos en ambos escenarios, gestión de la estabilidad y gestión de la incertidumbre. El primer escenario, se construye a partir del Plan Estratégico y del Reglamento de Estudios para Graduados de la Universi-

\footnotetext{
Fecibido: 00-06-09. Aceptado: 00-11-07
}

Arquitecto (LUZ), Master en Filosofía, en Planificación Urbana y Regional en la Universidad de Newcastle, Inglaterra, (1971-1973). Protesora Investigadora Titular de la Universidad del Zulia, Directora de Extensión durante el periodo 1996-1999 y, Coordinadora del Programa Académico de Especialización en Gestión Urbana, desde 1996 a Marzo del 2000. Ha participado como ponente en diversos Congresos Nacionales e Internacionales y es autora de varios artículos publicados en revistas arbitradas. Cursa actualmente el Doctorado en Ciencia Politica. E-mail: mferrer_98@yahoo.com o e.mall: mferrer@cantv.net. 
dad del Zulia, LUZ y, el segundo, a partir del Programa de Especialización en Gestión Urbana, primer postgrado acreditado de la Facultad de Arquitectura y Diseño de la misma universidad, que asumimos como propuesta emergente y pionera porque da respuesta a las determinantes del contexto, reconoce el alto potencial de los postgrados como plataforma generadora de conocimiento innovador, nueva teoría y praxis y, su filosofía se centra en la gestión y marketing del $\mathrm{Cl}$, para lograr dos objetivos fundamentales, potenciar la rearquitectura de la universidad y viabilizar el crecimiento sostenido del pais y su inserción en el contexto global.

Palabras clave: Gestión del capital intelectual, marketing, postgrado, redes, rearquitectura, escenarios.

\section{Management and Marketing of Intellectual Capital: Strategies for Positioning Post-Graduate Programs in the XXI Century}

\section{Abstract}

The global-digital context, networks and real virtuality, and the unprecedented crisis that the country is going through, require innovative answers from universities, and the redefinition and flexibilization of post-graduate programs, where management and marketing of intellectual capital assumes a strategic role. After recognizing this problem, the objective of this article is to answer four questions: ¿What characterizes the globalized relational world today, and what impact and demands for general change are generated by universities and postgraduate programs?; ¿What is intellectual capital (IC) and how is it managed towards empowering innovative capacities?; ¿How is knowledge managed in a complex world? ¿How does the university transform itself from the universituy of social compromise to a university of social revenue? The answers are formulated on the basis of a comparative analysis of two university and postgraduate management scenarios or options, the one which exists and the one which should exist, a functional or a tendencial scenario of the university dedicated to excelence and social commitment, and the emerging scenario of website postgraduate programs, internationaly recognized, that manage and market the intelectual capital of the university of social revenue of the third milenium. Management models are identified in both scenarios, the management of stability and the management of uncertainty. The first scenario is developed on the basis of a Strategic Plan and the Norms for Graduate Programs in the University of Zulia LUZ, and the second one is based on the Sepecialization Program for Urban Management, the first accredited postgraduate program in the Arquitecture and Design Faculty at the same university, which we accept as an emergent and pioneering program since it responds to contex- 
tual determinants, acknowledges the high potential of the postgraduate program as a generational platform for innovative knowledge, new theories, and practices, and a philosophy centered on management and marketing of $\mathrm{IC}$, in order to reach two fundamental objectives, to empower re-architecture in the university and to make viable sustained growth in the country and also its insertion in the global context.

Key words: Intellectual capital management, marketing, postgraduate programs, networks, re-architecture, scenarios.

\section{Introducción}

Iniciándose el tercer milenio, en la era digital y en un mundo globalizado, de redes y virtualidad real, en continuo cambio $y$, de gran incertidumbre por la velocidad de estos cambios, la gestión y el marketing del capital intelectual y del conocimiento innovador asumen un rol estratégico para lograr el crecimiento sostenido de los paises.

En este contexto, la universidad y los estudios de postgrado siguen anclados al pasado y, amarrados a principios y prácticas que refuerzan la lógica burocrática y la praxis politica universitaria, clientelar, jerárquica y autoreferenciada, cerrada a las demandas del país y del contexto, incapaz de gestionar la incertidumbre y de aprovechar la oportunidad que ofrece el contexto global. La universidad al igual que el pais, se debate entre grandes contradicciones, ahora más evidentes que nunca en esa convergencia insostenible, por un lado el populismo clientelar y, por el otro, la presión globalizadora y modernizante que exige por una parte a la universidad, eficiencia, calidad de la enseñanza, productividad y, alta rentabilidad social, lo que lógicamente resultará en alta rentabilidad económica para el país y, por la otra al Estado, una economía abierta al mercado.

Es una profunda crisis de mutación, que cuestiona su filosofía y actuación. En una crisis que se produce por la disonancia entre instituciones que no cambian y, las aspiraciones de la gente, cuyos hábitos si progresan, debemos como dice Touraine (1999), liberarnos de las dicotomlas artificiales, competitividad económica versus protección social y construcción europea o, latinoamerica en nuestro caso, versus identidad nacional, absoluto Estado versus absoluto mercado $y$, avanzar hacia una TERCERA VÍA o social liberalismo (Blair, 1998; Bresser, 1998; Giddens, 1999) o, hacia lo que propone como, la VÍA DOS Y MEDIO, vía intermedia entre la antigua social democracia y la tercera vía.

Este contraste entre la realidad global, dinámica, turbulenta $y$, cambiante a gran velocidad, que incluye $y$ excluye a paises $y$, donde los elementos clave son, según Crozier (1996), "la capacidad humana y tecnológica para generar, procesary producir conocimiento innovador" $y$, el arraigo de algunos sectores de la universidad y del pais, a una lógica y praxis burocrática y aislacionista que responden a una visión estable del mundo, hace que 
el país, la universidad y la oferta de estudios para graduados este descontextualizada y desactualizada, debido entre otras cosas a que, nos hemos excluido de la dinámica del mundo contemporáneo, (global, complejo, relacional, interdependiente, de redes), estamos ausentes ante la crisis del pais y ante la oportunidad que ofrece el proceso de cambio que enfrenta Venezuela y, somos cogestores y corresponsables del atraso que caracteriza a nuestro país, por cuanto reproducimos el modelo populista clientelar.

Por otro lado, el proceso de actualización del sistema educativo no va al mismo ritmo que el cambio tecnológico que demanda conocimiento innovador, nuevos enfoques y actitudes mentales. Esta situación provoca según Castells (1999), "dificultad para adaptarse a los nuevos sistemas de funcionamiento y genera personas que no saben tomar decisio. nes, con poco criterio y visión global y, 10 que es más importante, con poca autoestima" e, impide a la universidad y a los postgrados, adoptar una nueva filosofía académica centrada en lo que es medular de la universidad, LA GESTIÓN Y EL MARKETING DE SU CAPITAL INTELECTUAL en un ambiente flexible y plural, para lograr tres objetivos fundamentales: potenciar el conocimiento existente en ella, reforzar la solidez de una sociedad abierta al mundo y, viabilizar los cambios necesarios para lograr el crecimiento rentable y sostenido del pais $y$, su inserción en el contexto global.

En este sentido y, ante la crisis sin precedentes que atraviesa el pais, caracterizada según algunos autores, por el sobredimensionamiento de los factores subjetivos políticos sobre los factores reales de la economía, la caida de la economía en un $7,2 \%$ en 1999 (cifras del Banco Central), el desempleo ubicado entre el $15 \%$ y el $20 \%$, la economía informal que supera el $50 \%$ de la población activa y por un $75 \%$ de PYMES quebradas, son necesarias respuestas innovadoras de la universidad y de los estudios de postgrado. Para lograr este objetivo planteo tres propuestas básicas: la primera, REDEFINIR y FLEXIBILIZAR el postgrado para adecuarlo a las demandas del contexto; la segunda, asumir la gestión y el marketing del capital intelectual como estrategia para redefinir el rol de la universidad, de los postgrados y sus reglamentos; $y$, la tercera, se refiere a pasar de la visión funcional y de la práctica burocrática de "la universidad de la excelencia con compromiso social", (LUZ, 1998), al postgrado postburocrático, abierto, flexible, en red y homologado internacionalmente de "la universidad emergente con rentabilidad social del tercer milenio" (Ferrer, 1999).

La universidad rentable socialmente, produce beneficios sociales y económicos a la sociedad, reversibles a la academia e, impacta positivamente el medio, a través de egresados de pre y postgrado, investigación y extensión, de calidad, que soluciona problemas y construye oportunidades $y$, de esta forma, pasa a formar parte del sector productivo del país, porque asume la innovación/creación y difusión del conocimiento (básico y aplicable) como recurso renovable e inagotable de la universidad, impulsor de la economia y creador de riqueza 
(Toffler, 1997). En este sentido, Touraine plantea que, en el momento actual hay que orientar la enseñanza hacia la innovación. Esta nueva universidad promueve "el potencial de la sociedad para organizarse, capital social" (Fukuyama, 1996) $y$, recupera la confianza de la sociedad porque asume su responsabilidad ante el pais. Es en definitiva una universidad que, ante la exclusion se incluye y apoya al pais en su inserción inteligente en el mundo global, que ante la ausencia hace presencia activa y decisiva y, que ante la praxis del todo vale, es rentable socialmente, donde los resultados cuentan. Para Guédez (1998),"presencia significa lo contrario de ausencia, es sinónimo de vida y ausencia es expresión de muerte. Estar presente significa asumir responsabilidades, afrontar $y$ actuar $y$, ausencia significa evasión, huida, deserción".La presencialidad implica, "una educación atenta al devenir y sintonizada con las exigencias derivadas de un mundo sometido a permanente cambio".

El objeto de este trabajo es analizar los estudios de postgrado desde la perspectiva de la gestión y el marketing del capital intelectual, que asumo como recurso inagotable de la universidad con rentabilidad social y para ello respondo brevemente cuatro preguntas: ¿Qué caracteriza al mundo global - relacional de hoy y, qué impacto y demandas de cambio genera, en la universidad y en los estudios de postgrado? ¿Cómo se gestiona el conocimiento en un mundo complejo? ¿Qué es y cómo gestiona la universidad su capital intelectual (Cl) para potenciar las capacidades innovadoras? ¿Cómo pasar de la universidad del com- promiso social a la universidad con rentabilidad social?

Para responder estas preguntas analizo dos escenarios $u$ opciones de gestión de la universidad y de los estudios de postgrado, de lo que es y de lo que debe ser, el escenario funcional o tendencial de la universidad de la excelencia con compromiso socialy, el escenario emergente del postgrado en red $y$, homologado internacionalmente, que gestiona y mercadea el capital intelectual de la universidad con rentabilidad social del tercer milenio e, identifico, los paradigmas implicitos en ambos escenarios. El primer escenario, lo construyo a partir del Plan Estratégico (LUZ, 1998) y del Reglamento de Estudios para Graduados de LUZ (1998a) y, el segundo, a partir del Programa de Especialización en Gestión Urbana, PEGU, primer postgrado acreditado de la Facultad de Arquitectura y Diseño de LUZ, FADLUZ, que asumo como propuesta emergente porque da respuesta a las determinantes del contexto, reconoce el alto potencial de los estudios avanzados como plataforma generadora de conocimiento innovador, nueva teoria y praxis $y$, su filosofia se centra en la gestión y marketing del conocimiento para lograr dos objetivos fundamentales, potenciar el capital intelectual de la universidad y viabilizar el crecimiento sostenido del país y su inserción en el contexto global.

Siven de antecedentes a este articulo los planteamientos que realicé con anterioridad en las ponencias, "Los Estudios de Postgrado en un contexto Global, una visión prospectiva" (Ferrer, 1997) y, "Justo a tiempo el PEGU entró en agen- 
da" (Ferrer, 1998), en los cuales analizo el futuro del postgrado y de la Especialización en Gestión Urbana y propongo cambios al Reglamento de Estudios para Graduados de LUZ (1998a) y en la gestión de los Trabajos Especiales de Grado de dicha Especialización.

\section{Contexto y conceptos básicos: el conocimiento en un mundo complejo}

Cinco aspectos clave sirven para describir el contexto y, los conceptos que enmarcan este artículo: gestión de la estabilidad, gestión de la incertidumbre, un mundo relacional-comunicacional de redes y virtualidad real, gestión y marketing del capital intelectual, global-local y gestión urbana.

\subsection{El conocimiento en un mundo complejo}

Guédez (1998), entiende por contexto "el espacio geopolítico y el tiempo histórico que establecen el marco de desenvolvimiento socio-educativo". Para este autor la realidad actual tiene tres características básicas: disruptividad, impredecibilidad y complejidad. La disruptividad se presenta como turbulencia vinculada al cambio, el cual es cada vez más rápido en su ritmo, más profundo en su contenido y, más global en su expansión. La impredecibilidad, se concreta en el surgimiento de desenlaces inesperados, lo que ha llevado a sustituir las palabras destino y porvenir por devenir, donde el futuro es un mercado libre de oportunidades que se renuevan conti- nuamente en el presente y, la complejidad, hace que los acontecimientos en la realidad sean multideterminados y plurideterminantes, ahora nada se comprende sin relación con un todo, ya que ningún fenómeno responde a una sola causa.

Estos aspectos se unen para configurar un ambiente psicosocial caracterizado por la incertidumbre y la paradoja, según Guédez (1998). Para este autor, la incertidumbre es el resultado de la ausencia de síntesis totalizadoras y, la paradoja, es la presencia y, coexistencia de situaciones contradictorias. Ante esta dinámica han surgido nuevos enfoques para abordar, analizar y actuar sobre la realidad, que para Guédez (1999:210), son los siguientes: presencialidad (atención sostenida, asumir un posicionamiento ante la realidad); apertura (implica la ampliación de los márgenes perceptivos y el fomento del pensamiento divergente); flexibilidad (plasticidad que proporciona el pluralismo contrario a todo esquema totalitario); creatividad-innovación (traducción práctica de una presencia abierta y plural, a favor de resultados eficaces y eficientes) y, eticidad (ejercicio integro y auténtico apoyado en valores morales).

Guédez identifica cinco impactos/demandas de estos nuevos paradigmas en la educación: La presencialidad, demanda una educación atenta al devenir y a un mundo en permanente cambio. La apertura, requiere una educación sensible a la globalización y descentralizada. La flexibilidad, demanda una educación plural y la promoción del pensamiento divergente, fundamentado en una continua actualización de las preguntas. La innovación promueve la educación ejercida 
desde la búsqueda y creación de conocimiento innovador. La eticidad, demanda una educación inspirada en valores morales y sociales. Como aspectos complementarios a estos aspectos contextuales Guédez (1998), plantea que, "los valores no están enterrados, los ideales no han muerto y, la historia no ha terminado, el futuro no está previsto dentro de una determinada perspectiva, es el resultado de lo que seamos capaces de hacer aqui y ahora, es decir, es acción en el presente, es ejercicio de una presencia, es encrucijada de posibilidades".

\subsection{Un mundo postburocrático, flexible y plural}

Desde una perspectiva dinámica, Goma y Brugué (1998), identifican dos modelos de gestión: el modelo de gestión de la estabilidad y el modelo de gestión de la incertidumbre. El primer modelo de gestión de la estabilidad, se ubica temporalmente entre la finalización de la segunda guerra mundial y los 70 , aunque su influencia llega hasta nuestros dias. Durante este periodo hay un crecimiento estable de la economía, se consolida el estado de bienestar, se aumenta la capacidad de regulación y provisión pública y se adopta un consenso estratégico entre las fuerzas politicas. Este modelo de gestión se caracteriza por la autosuficiencia (monopolio en la prestación de los servicios-organización cerrada), la uniformidad (ofrece productos estándares para todos), el profesionalismo (gestión dominada por expertos que imponen fuertes divisiones funcionales, la segmentación artificial de la actividad administrativa, ocasionando pérdida de visión estratégica de los problemas), paternalismo y monopolismo, que refuerzan las lógicas estandarizadoras y universalizadoras, una respuesta igual para todos. La gestión de la estabilidad se define a partir de unos objetivos claros, invariables y prefijados que condicionan fuertemente las estructuras organizativas. El resultado es una compleja organización, generadora de economías de escala, pero también de fuertes inercias y rigideces.

Según estos autores, a partir de mediados de los 70 , el modelo de gestión de la estabilidad entra en crisis debido a las transformaciones del entorno socioeconómico y político, el cuestionamiento al Estado de Bienestar y, la ruptura del consenso entre las distintas fuerzas políticas. Estas transformaciones, que revolucionan la producción de bienes y servicios (postfordismo), introducen nuevas demandas de descentralización, especialización, pluralismo y flexibilidad organizativa, para manejar un entorno cada vez más complejo y cambiante. Al desaparecer la estabilidad $y$, la continuidad, aparece el nuevo modelo de gestión de la incertidumbre. Según la hipótesis de Goma y Brugué, actualmente se estaria sustituyendo el primer modelo por el segundo, lo cual evidencia la obsolescencia de la organización burocrática tradicional, caracteristica predominante del Estado Venezolano y de la universidad.

El segundo modelo de gestión de la incertidumbre, según los mencionados autores, supera el concepto de gestión entendida como dirección de sistemas rígidamente organizados con responsabili- 
dad claramente definida y con objetivos precisos y, se sustituye por un nuevo concepto de dirección que, con una renovada capacidad de innovación y creación, desarrolla políticas capaces de movilizar redes de apoyo que garantizan el éxito de su implantación, fomentando un estilo de gestión que refuerza los componentes directivos, que es más proactiva que reactiva y más promotora que indicadora.

Los principios de la gestión de la incertidumbre, según Gomá y Brugué son cuatro: pluralismo (organización vista como constelación de organizaciones con recursos, capacidades y limitaciones); diversidad ( refuerza y mejora los canales de comunicación y las fórmulas de participación); cambio (el contexto actual se caracteriza por la turbulencia y la incertidumbre, las necesidades y soluciones cambian rápidamente); dirección estratégica (la gestión de la incertidumbre requiere de capacidades globalizadoras para abordar los problemas en toda su complejidad, ya que las divisiones funcionales generan rigidez contraria a la flexibilidad que demanda la incertidumbre). La gestión de la incertidumbre asume dos modalidades según Goma y Brugué (1998): gestión de la influencia y gestión del cambio. La primera, conecta los principios de diferencia y piuralismo y responde a la necesidad de resituar la gestión en redes más complejas de relaciones interorganizativas en que se desarrollan actitudes de negoclación, coordinación y promoción. La segunda, la gestión del cambio, responde a las exigencias de flexibilidad, especialización y de dar res- puestas creativas e innovadoras en un entorno en constante transformación.

\subsection{Un mundo relacional- comunicacional, de redes y virtualidad real}

Según Crozier ( 1996), en la sociedad actual donde, la mayoría de las actividades humanas se vuelven inmateriales y relacionales, la comunicación y el recurso humano, son aspectos clave. Para Crozier, la sociedad en respuesta a esta nueva realidad, se organiza en redes interactivas, para aprender mediante el intercambio y la cooperación y, de esta mane$\mathrm{ra}$, hacer frente mejor a los nuevos retos relacionados con mayor libertad, mayor complejidad y, mayor capacidad relacional, en un contexto mucho más pluralista de actores, intereses e interacciones. En este sentido, Goma y Brugué (1998), plantean que, "es la actitud relacional la que permite influir en la pluralidad".

Por otro lado, Castells (1998a:3), plantea que el sistema global tiene estructura de red que, "valiéndose de la flexibilidad proporcionada por las tecnología de la información (la era digital de Gates), conecta lo que vale y, desconecta lo que no vale o se devalúa, personas, empresas, territorios $y$ organizaciones". Para este autor, los Estados, además de aprender el arte de navegar en los flujos, que sustituye el ejercicio del poder soberano como forma de gobierno, están respondiendo a la globalización organizándose en red que significa compartir autoridad, o sea, la capacidad de imponer una decisión. La red, se configura sin centro, a través de nodos interconectados, de di- 
ferentes dimensiones, donde las relaciones son frecuentemente asimétricas, pero donde todos los nodos son necesa. rios. La red, está hecha de negociación y decisión, de compromiso y autoridad, de información y estrategia, y conecta al Estado nación con los niveles supranacionales y subnacionales, locales, regionales, institucionales y ONGs. Según Castells, el funcionamiento en red de relaciones, asegura la coordinación y la descentralización en la misma organización compleja y, es un potencial de la era de la información. A partir de estos planteamientos Castells (1998a:4) conceptualiza la empresa red, como "nueva forma de organización y gestión, o sistema operativo efectivo, que asume la productividad y la competitividad como procesos clave de la economía informacional - global, en la cual, la productividad proviene de la innovación y la competitividad de la flexibilidad". Por otro lado y, según Castells (1999), el trabajo en red potencia la flexibilidad de las empresas y la adaptación rápida a una demanda en continuo cambio. Para Guédez (1998), "el pluralismo y la flexibilidad son expresiones teóricas de la innovación y la creatividad".

Gates (1999), plantea que el desarrollo de una "lógica en redy, de nuevas capacidades de asociación y conexión, viabilizan la aparición de un estilo de trabajo en red $y$, un estilo de vida en red". EI ESTILO DE VIDA EN LA RED, según Gates (1999), "cambia la relación empresa consumidores, administración - ciudadanos $y$, en último término pone el control de la relación en manos del consumidor ciudadano". En el mundo contemporáneo, relacional, de flujos, redes y virtuali- dad real, para Gates, "Ia conectividad cobra un significado más amplio que poner en relación dos o más personas, ya que la RED crea un nuevo espacio universal de información compartida e introduce un factor completamente nuevo, la capacidad para hallar información y para agrupar personas con intereses comunes". En este sentido y, según Castells (1998), "la cultura de la virtualidad real, se construye, en la era informacional. global, alrededor de un universo audiovisual cada vez más interactivo que integra a las diversas culturas en un hipertexto electrónico".

\subsection{Capital Intelectual y Gestión del Conocimiento}

Para Gates (1999: 276), el Capital Intelectual no es sólo un concepto funcional, "es el valor intrínseco de la propiedad intelectual de una compañía y del conocimiento que posee el personal". Administrar correctamente ese capital eleva el $\mathrm{Cl}$ corporativo y, toda empresa para tener éxito necesita tener un Ci corporativo alto, "lo cual tiene una repercusión directa en la valoración de la empresa $y$, en su permanencia en el mercado", (Gates, 1999). Por otro lado, la gestión del conocimiento, para Gates, es un aspecto clave que debe incluirse en los objetivos y procesos de la empresa, a partir del reconocimiento de la necesidad de compartir información. En este contex to, la información es un medio y no un fín, el fin es crear una inteligencia institucional, lo que Gates llama el Cl corporativo. Para Gates (1999:277), "el Cl corporativo es la medida de la soltura con que 
Gestión y marketing del capital intelectual Ferrer y Arroyo, Mercedes

la empresa distribuye la información al más alto nivel y de cómo las personas de la institución pueden continuar desarrollando sobre las ideas de otros y, compartir el conocimiento histórico y el actuar. Para que la gestión del conocimiento sea exitosa hay que crear un ambiente que estimule la colaboración y el conocimiento compartido, que es un concepto clave que debe ser asumido por la alta dirección de la empresa y por ende por la universidad. Los ambientes que favorecen la discusión, prospera la iniciativa y las responsabilidades personales. En este sentido Crozier (1996:37) plantea que, "hay que pasar del mundo de la obediencia al mundo de la responsabilidad" $y$, que, "el mundo postindustrial, se dirige cada vez más a lo relacional y, exige sino trabajar en grupo, al menos en cooperación".

La gestión del conocimiento, según Gates (1999:300), "no es más que un término de moda para una idea sencilla, lo que se gestiona en realidad son datos, documentos y el trabajo de las personas (flujos de información y el conocimiento), con la finalidad de mejorar la forma en que las personas colaboran, comparten ideas, a veces discuten $y$ otras veces construyen sobre ideas ajenas $y$, poner todo eso en concierto con vista a un designio común". También podemos concebir la gestión del conocimiento "como una inversión en capital intelectual (Cl), que con el tiempo conduciráa un $\mathrm{Cl}$ corporativo más alto, es decir, incrementará la capacidad de la empresa para concitar el mejor pensamiento y la mejor acción en el plano colectivo" (Gates, 1999:296).
Estos cambios están transformando el trabajo, generando impactos y nuevas demandas en los estudios de postgrado, entre estas la internacionalización de las profesiones y la globalización del mercado de trabajo de la mano de obra más cualificada. Según Castells (1999: 33), "una persona que empiece su vida profesional ahora, a lo largo de su vida cambiará de profesión más o menos cuatro veces" $y$, "las personas que sean capaces de redefinir lo que tienen que hacer, de volver a aprender para saber como hacer las nuevas tareas, nunca quedarán obsoletas". En este sentido, este autor plantea que, "el fundamentalismo cartesiano esta superado, es demasiado lineal y causale impide comprender y razonar dentro de lo complejo", por lo que es necesario REDEFINIR EL SISTEMA EDUCATIVO $y$, los estudios de postgrado, que deben viabilizar según Castells (1999:39), "el desarrollo de la capacidad social para hacer transferencias, entre el trabajo y la educación contantemente". En este sentido, Crozier (1996:37), plantea que, "el aprendizaje del dominio de la complejidad y, la capacidad de reducirla, sólo puede llevarse a cabo mediante una nueva pedagogía de los conocimientos, la velocidad del cambio exige reeducación continua y asumir la formación como proceso para toda la vida".

\subsection{Marketing del Capital Intelectual y del Conocimiento}

Ante esta realidad, algunas universidades nacionales perciben la globalización como oportunidad para reforzar sus 
ventajas competitivas y para asumir nuevos roles. La universidad debe, en este contexto, plantearse un manejo distinto del gobierno universitario y de su gobernabilidad, incorporándose activamente y liderizando el proceso de reestructuración académica y administrativa para adecuar la educación a los nuevos retos locales y globales. En este contexto, los estudios de postgrado asumen un decisivo rol y, surgen como espacio privilegiado tanto para apoyar, a través de la formación de IV y $\vee$ nivel, el crecimiento económico - social y competitivo del país como para articular políticas académicas globales y locales. Esto requiere la adopción de nuevos paradigmas por parte de las Universidades para modernizar y mejorar los procesos de gestión del conocimiento y el mercadeo de los estudios de postgrado y adaptarlos al contexto global (homologarlos) en función de viabilizar el desarrollo sostenido y equitativo del país.

Este planteamiento considera los cambios tecnológicos, políticos y económico - sociales, sucedidos en los últimos años, que están impactando tanto al país como a las Universidades, entre los que destacan seis como fundamentales: la globalización, que combina competitividad, liberalización y flexibilización y el desarrollo de redes de relaciones y de comunicación; la internacionalización de las profesiones para hacerlas homologables a nivel mundial; la cooperación entre paises culturalmente afines que según Huntington (1997), genera el marco donde prospera un nuevo orden mundial basado en las civilizaciones, en e! cual las sociedades que comparten afinidades culturales crean redes de coopera- ción y de solidaridad. El nuevo protagonismo de las Universidades, que se articulan en redes de cooperación internacional, con creciente capacidad de lobying que les permite posicionarse en el mercado de la educación superior, el conocimiento y el $\mathrm{Cl}$ como impulsor de la economía y creador de riqueza en el mundo global y, la nueva economia digital basada en la innovación y el cambio (Verwaayen, cit. por Fernández, 2000; Lewis, 2000). En respuesta a estos cambios se identifican dos tendencias que coexisten, la creciente demanda de I+D o investigación aplicada ("problem solving") $y$, el desarrollo de investigaciones en red con dos objetivos, crear y desarrollar conocimiento innovador $y$, sustentar y apoyar postgrados en red o globalizados, para mejorar la competitividad y, el crecimiento sostenido de los países ya que, según Touraine (1999), "es el momento para volver a poner el acento sobre la innovación, la educación y la solidaridad, ya que no existe posibilidad de crecimiento sostenido sin innovación".

Según Verwaayen (en Fernandez, 2000), para crear prosperidad, "la innovación y la creatividad son la clave del éxito" $y$, todo lo que se necesita para participar en la nueva economía digital, "es acceso a las redes de comunicación $y$, en este sentido, ninguna nación o región esta excluida, lo importante es encontrar vías para dominar y utilizar la información". Para los gobiernos los retos se concentran en impulsar una educación eficiente $y$, de esta manera aprovechar los cambios y disminuir la brecha entre los que tienen y los que no tienen, acceso a la información y, apoderarse de las oportuni- 
dades. Por otro lado $y$, ahora que los negocios y las universidades y postgrados no tienen fronteras geográficas, el mayor reto está en romper todas las reglas del juego, crear nuevas estructuras, mejor diseñadas para las necesidades cambiantes $y$, prepararse para resolver conflictos entre los canales de distribución y la red. Según Lewis (2000), los Teóricos del Nuevo Crecimiento, desde hace casi una década, han planteado que la innovación no es otro factor más en el crecimiento, sino el más importante de todos. La fe en la importancia de la innovación es la base del gran cambio generado en las actitudes hacia la tecnologia y, de los emprendedores responsables de convertir tecnología en negocios.

Según Lewis (2000), con el auge de Internet, las ideas de los Teóricos del Nuevo Crecimiento, han influido las mentes de los políticos que han asumido la aparición de una nueva economía basada en la innovación y el cambio. Según Romer (en Lewis, 2000), "la riqueza es simplemente otra forma de llamar al cambio". La economía digital que ha aparecido con la red, ha traído numerosos cambios en la forma de vida en el mundo occidental en general, algunos de ellos han sacudido los cimientos de la economía, otros son movimientos más sutiles que afectan las actitudes básicas de la gente. Muchos de estos cambios según Lewis (2000), serán perdurables, incluso si el auge es una burbuja y si la burbuja explota mañana, entre estos destacan dos como fundamentales, el nacimiento y potenciación del nuevo emprendedor - fundador de empresas en la red que asume riesgos continuamente $y$, la democratiza- ción del capital. Según este autor, cuando el capital se democratiza se desmitifica y, se convierte en un producto básico, accesible a todos, en teoría, lo cual conlleva otros cambios sociales que han recibido un gran impulso con el Internet. Browning y Reiss (2000), plantean dos constantes en el mundo actual en continuo cambio, "las enormes nuevas oportunidades que genera la nueva tecnología y la destrucción creativa del capital, siendo la clave de la nueva economía el crecimiento". Otros autores como Samuelson (2000), entre otros, contradicen esta visión optimista y, plantean los riesgos de la economía digital, "es preciso controlar la burbuja de Wall Street, no hay que dejarse llevar por el pesimismo del fin del mundo ni por una fe estática en el boom de una nueva era". Para Byron (2000), "la alucinación en masa inspirada por compañías en Internet sobrevaluadas, augura días bastante oscuros".

La nueva economía digital, el surgimiento de redes de cooperación y alianzas estratégicas se enmarcan en la hipótesis de Huntington (1997) que establece que, la cultura y las identidades culturales, que son en su sentido más amplio identidades cilivizacionales, están configurando las pautas de cohesión, desintegración y conflicto en el mundo de la postguerra fria. A partir de esta hipótesis central Huntington (1997) plantea que la politica global es a la vez multipolar y multicivilizacional, donde la modernización económica y social no esta produciendo ni una civilización universal ni la occidentalización de las civilizaciones no occidentales. Lo que esta surgiendo "es un nuevo orden mundial basado en las ci- 
vilizaciones a partir del cual las sociedades que comparten afinidades culturales cooperan entre si". Esto explica la tendencia prevaleciente a la cooperación entre países y, el interés creciente de España y Europa por lberoamérica y de Estados Unidos por la integración de las Américas. Los bloques surgen a partir de la afinidad y complementariedad cultural generando solldaridades entre países culturales afines y programas de cooperaciốn internacional entre paises y Universidades. Este nuevo escenario donde prospera la solidaridad y la cooperación $y$, promueve el intercambio de conocimiento entre universidades (potencial de la era digital), es el resultado tanto de la actividad paradiplomática desarrollada por las universidades como por la asistencia de profesores a Congresos Internacionales y la comunicación a través de la RED, que viabiliza el contacto con otros centros de estudio $y$, el intercambio de ideas y proyectos, dirigidos a la creación de redes de investigación y postgrados a partir de alianzas estratégicas entre Universidades Nacionales y Extranjeras.

La globalización implica a nivel universitario competir, cooperar y co. nectarse, aplicando políticas de marketing de su Cl para lograr la visión y, mejorar las condiciones académicas, todo lo cual conlleva a una mayor eficacia y productividad universitaria. La competitividad fortalece la especialización, de esta forma las universidades deben concentrar sus producciones en aquéllas áreas en las que son más competitivas a nivel nacionale internacional. Por otro lado, no es sólo la ventaja competitiva la que afirma la integración al modelo de globaliza- ción, sino fundamentalmente las ventajas comparativas, entre las que destacan la eficiencia de las universidades para pres. tar los servicios que requiere la globalización: la gestión (en sentido amplio) del capital intelectual, que planteo como el proceso mediante el cual se crea, adapta, divulga y aplica, conocimiento innovador que soluciona problemas y construye oportunidades. Actualmente algunas universidades rentables socialmente, que asumen su responsabilidad ante el país, rivalizan por obtener un espacio propio mediante la identificación de sus puntos fuertes y la promoción de su imagen, la lucha por el reconocimiento (Fukuyama, 1994). En este sentido y, para atraer recursos, ponen en marcha proyectos para la región y el país como, parques tecnológicos, empresas rentales, y alianzas estratégicas, entre otros, dirigidos a mejorar la calidad de vida e insertarse en la economía mundo. Las políticas de mercadeo universitario, a nivel nacional e internacional, están dirigidas a potenciar las ventajas comparativas de cada universidad y. su potencial de innovación, dentro de un esquema diferenciado de ofertas especializadas, a través de su integración a redes de universidades.

En este sentido, Kennedy (1993), plantea que existen dos formas de actuación con respecto a las reformas que hay que acometer si una sociedad decide mejorar su actuación frente al siglo XXI, el laissez - faire (dejar hacer, dejar pasar, actuación típica de la universidad) o, prepararse en forma activa para el futuro. Las respuestas no van a ser uniformes, sin embargo la realidad exige a la Universidad asumir el reto de preparar a la so- 
ciedad para el futuro que es ahora, ya. En este contexto, la Universidad rentable socialmente asume un nuevo rol, que implica cambios en sus presupuestos filosóficos y prácticos, para dar respuesta a los nuevos desafíos de la globalización ya que, la tecnología emergente crea puestos de trabajos nuevos y elimina viejos, esto demanda un sistema nacional de formación y reeducación continua y requiere la alianza estratégica y la cooperación entre tres actores clave: Universidad, Empresas y Gobierno. La educación significa algo más que reequipar la fuerza de trabajo, implica un profundo conocimiento de por qué esta cambiando el mundo y de lo que tenemos en común como sociedad global, entre otros aspectos. Los desafíos tecnológicos, las cuestiones de género, la emigración, el medioambiente, el impacto de la globalización en los valores y la cultura son temas de interés en todas partes del mundo que exigen respuesta (Kennedy, 1993). Esto supone desafiar intereses creados, alterar hábitos viejos, corregir estructuras y construir una VISIÓN COMPARTIDA DE FUTURO en una época que pocos gobiernos y universidades son capaces siquiera de enfrentarse al corto plazo. Esto implica confrontar el rechazo y asumir el riesgo porque la mayoria se resiste al cambio y, demanda, una universidad posicionada en la historia, pluralista, innovadora y proactiva.

\subsection{Global, Local y Gestión Urbana}

La globalización, según Castells (1998a:2), "es un proceso históricamente nuevo, distinto a la internacionalización y a la existencia de la economía mundial, porque solo en la última década se ha constituido un sistema tecnológico (comunicaciones, sistemas de información interactivos, transporte de alta velocidad de personas y mercancias) que hace posible la globalización". En este contexto, el Estado nación se encuentra atrapado entre, las exigenclas contradictorias de la operatividad global y, la legitimidad nacional (demandas ciudadanas). Para enfrentar esta contradicción los gobiernos, según Castells (1998), han emprendido un vasto esfuerzo de descentralización, dirigido a conectar más directamente identidades e intereses con instituciones políticas, como paso previo a la articulación de los distintos niveles institucionales en una red compleja de conexión entre lo local y lo global. El estado se relegitima por la descentralización, (la local en un mundo globalizado) lo cual se constituye en una de las paradojas de este tiempo, el relanzamiento de lo local en la era de lo global. Los gobiernos locales presentan mayor flexibilidad para adaptarse y negociar con los flujos globales, tienen una relación más fluida con los ciudadanos $y$, en consecuencia, mayor capacidad de intervención en red y de forma coordinada con los distintos ámbitos de gobierno del territorio. La proximidad entre el gobierno local y los ciudadanos permite un control social más transparente, refuerza la oportunidad de participación política y de relegitimación del Estado.

En este contexto, la gestión urbana es el conjunto de procesos dirigidos a operar sobre la ciudad e implica la articulación, en lógica de red, de recursos humanos, financieros, organizacionales, 
políticos y naturales, para construir y mantener la ciudad y satisfacer las necesidades individuales y colectivas de los distintos agentes sociales y, de conexión con los ámbitos nacionales, regionales e internacionales (CEPAL, 1995). Según la CEPAL, seis supuestos enmarcan la gestión urbana moderna: la gobernabilidad de la ciudad, una eficiente distribución de los recursos, el desarrollo local con equidad, el mejoramiento de la calidad de vida, la participación en la formulación de políticas y la descentralización. La gobernabilidad de la ciudad depende de la capacidad del gobierno local de definir su propio proyecto político y de articular y gestionar acciones para que se pueda concretar.

\section{Dos enfoques en tensión}

De los escenarios de futuro de los ESTUDIOS DE POSTGRADO, analizo dos escenarios, el escenario funcional - tendencial, representado por el Plan Estratégico y, en el Reglamento de estudios para graduados de LUZ (REPG), $y$, el escenario emergente o imagen objetivo de los estudios para graduados que demanda el mundo actual, representado por el Programa de Especialización en Gestión Urbana. El análisis se realiza contrastando nueve variables que definen y caracterizan los escenarios y, los paradigmas de organización, que subyacen en estos escenarios, el paradigma burocrático de la gestión de la estabilldad y el paradigma postburocrático de gestión de la incertidumbre. El análisis de escenarios tiene como objetivo según Francés (1998:4), "flexibilizar los mode- los mentales de quienes toman decisiones e identificar las principales opciones de lo que podría pasar".

\subsection{Escenario funcional}

El escenario funcional se define a partir de un modelo de universidad entendido como: INSTITUCIÓN DE EXCELENCIA ACADEMICA CON COMPROMISO SOCIAL, que plantea el Plan Estratégico de LUZ (1998) y el Reglamento de Estudios para Graduados de LUZ, REPG (1998a). Este plan, representa una propuesta de gestión estratégica a largo pla$z 0 y$, se instrumenta deliberadamente, a partir de ocho (8) valores institucionales, seis (6) objetivos estratégicos, cincuenta y una (51) estrategias institucionales, seis (6) fundamentos iniciales, dieciséis (16) principios éticos, nueve (9) programas direccionales, cuarenta y seis (46) proyectos estratégicos, tres (3) políticas académicas, formuladas a partir de las 3 funciones básicas, investigación, docencia y extensión y, cinco (5) políticas de apoyo académico. El postgrado aparece como uno de los tres grandes ejes de la docencia en el Programa Direccional 2. "Innovaciones Académicas".

En relación con los actores, el plan identifica tres (3) niveles de lideres a los que se les asigna roles diferenciados de acuerdo a una lógica burocrática, jerárquica, vertical y en cascada. En el primer nivel se sitúa el Consejo Universitario que "define las políticas universitarias $y$ directrices de su conducción, en el segundo, las Facultades, Núcleos y Dependencias Centrales, que direccionan los Iineamientos estratégicos del Plan, para 
garantizar su concreción y, en el tercero, "otras unidades organizativas que operacionalizan los lineamientos estratégicos, donde a partir del hacer universitario se avanza hacia el debe ser" (LUZ, 1998). "Este es un escenario cerrado, que sólo invitó a participar a los protagonistas tradicionales $y$, se olvidó del otro público universitario $y$, de la sociedad, que pudo tener algo que decir" (Goma y Brugué, 1998). En esta lógica y, escenario, la retroalimentación, el trabajo en red, la pluralidad, la descentralización y la democracia deliberativa y/o dialogante (Crozier, 1996; Guiddens, 1996), están negados.

En relación con el REPG de LUZ, del análisis comparado realizado por la autora de este articulo (Ferrer 1997), entre los postgrados de Venezuela (Universidades del Zulia y Central de Venezuela), España (Universidades de Valladolid y Valencia) y, Colombia (Universidad Nacional), destacan las siguientes conclusiones generales y especificas:

De la evaluación realizada de los postgrados (programas académicos) de los tres paises y las cinco universidades mencionadas anteriormente, considerando tres variables número de unidades crédito, número de materias y número de horas o dedicación, destacan las conclusiones generales siguientes: los doctorados Españoles exigen Unidades Crédito (UC), tiempo y número de materias más cercano a nuestras especializaciones $y$, a las maestrías de las universidades colombianas que a las maestrías de Venezuela, para todos los casos analizados. Los doctorados españoles no exigen como requisito de ingreso título de Espe- cialización o Maestría. En relación al caso colombiano, el número de materias, de UC y de horas se acerca más a la especialidad venezolana que a las maestrías y por lo tanto a las tres variables analizadas del Doctorado en España.

Si se considera que por tener poca densidad horaria, unidades crédito o materias, los doctorados españoles no tiene calidad o la profundidad requerida creo necesario destacar la importancia que reviste el doctorado en España, citando el Real Decreto 185/ 1985 del 23 de Enero que regula el tercer ciclo de los Estudios Universitarios en España según el cual, "el tercer ciclo (doctorado) constituye condición esencial para el progreso cientifico $y$, por ello para el progreso socialy económico de una comunidad, por cuanto de la profundidad de sus contenidos y seriedad de sus planteamientos depende la formación de los investigadores. Tiene además una consecuencia de extrema importancia, en él se inicia la formación del profesorado universitario, considerando que en la Universidad docencia e investigación son dimensiones inescindibles. Por ello el tercer ciclo es decisivo para promover la calidad de la enseñanza y para potenciar la investigación y, articular estas dos funciones universitarias".

Observamos en los postgrados venezolanos:

1) El potencial de homologación de los postgrados de LUZ es bajo. Las normas del Consejo Nacional de Uuniversidades (CNU), el Reglamento de Estudios para Graduados (LUZ, 
1998a), coloca de entrada en desventaja a los EPG de LUZ para integrar redes globales porque exige más unidades crédito (UC), materias y horas de clase que los Estudios para Graduados de España y Colombia (Ferrer, 1997).

2) La alta densidad horaria, de contenidos y de materias de los programas de LUZ, no son necesariamente indicadores de calidad, por el contrario su excesiva escolaridad presencial (clases magistrales) frustra a muchos profesionales que quisieran ingresar a estos estudios. Un análisis de deserción en postgrado 0 , de convalidación, podría confirmar esta hipótesis (Ferrer, 1997).

3) El diseño conceptual del Reglamento de los EPG no consideran el conocimiento como recurso inagotable de la universidad (Ferrer, 1998a), ni la gestión del capital intelectual (CI) de LUZ, como idea fuerza para direccionar los procesos propuestos. En consecuencia el potencial para promover la creación de un ambiente colaborativo y cooperativo que estimule la creación de una plataforma de conocimiento innovador y el trabajo en red, es muy bajo, es decir, queda a criterio personal del coordinador de la maestría o especialización, pero no como política institucional.

4) No existe una política de divulgación $y$, mercadeo del $\mathrm{Cl}$ de LUZ, que garantice competitividad y rentabilidad a los postgrados ni su impacto en la realidad local y global (Ferrer, 1997).

En sintesis se puede concluir que, el modelo universitario en general, la gestión de los postgrados en especial y su estructura organizativa, están fuertemente condicionados por una lógica burocrática y conformista muy difícil de cambiar, que funciona en base a la jerarquía, las reglas y el control que hace respetarlas, pero que al mismo tiempo paraliza su ejecución. En este escenario LUZ y las DEPG, siguen siendo complejas organizaciones, generadoras de fuertes inercias y rigideces. En definitiva $y$, contrario a su concepción estratégi$\mathrm{ca}_{1}$ el Plan Estrategico de LUZ y el REPG, asumen el paradigma burocrático de gestión de la estabilidad, que se caracteriza por una visión compartimentada en tres funciones básicas, docencia, investigación y extensión, por ser prescriptivos del "debe ser", reactivos y, donde el diseño de políticas se realiza bajo una visión jerárquica, con fuerte tendencia formalizadora y reforzadora de lógicas estandarizadoras y universalizadoras. Este paradigma es contrario a la necesidad de flexjbilidad y pluralidad que exige el contexto relacional, inviabiliza, el trabajo en redes $y$, la posibilidad de hacer aportes estratégicos a la transformación universitaria y a los postgrados. Por otro lado, el Plan y el REPG no se han diseñado desde la lógica de la gestión y marketing del $\mathrm{Cl}$ de la universidad, ni han considerando el conocimiento como "recurso inagotable de la universidad" (Ferrer, 1998a).

\subsection{Escenario emergente: La Especialización en Gestión Urbana}

Cinco son las claves o fortalezas, existentes y potenciales, del Programa de Especialización en Gestión Urbana, 
PEGU, que validan su presentación como escenario emergente $y$, que han viabilizado su acreditación, en menos de tres años desde su inicio en 1994 y, que por lo tanto pueden constituirse en criterios básicos para la acreditación de postgrados: 1. EIPEGU, se apoya en alianzas estratégicas que potencian la conformación de redes y la gestión y marketing del conocimiento, a través de tres aspectos fundamentales: a. la colaboración entre agentes sociales involucrados en la gestión urbana, b. compartir conocimiento entre instituciones y participantes, $y, c$. la articulación de la Investigación, con la Docencia y la Extensión, $(1+D+E=A c a d e-$ mia), 2. El planteamiento de homologación de la unidad crédito a los estándares latinoamericanos 3. la nueva propuesta de gestión de los Trabajos Especiales de Grado, TEG, que conlleva : su asunción como investigación de agenda y, la incorporación de estrategias docentes y operativas, entre estas, tres Seminarios donde se elaboran los TEG, con dos objetivos, eliminar el TMT (todo menos tesis) y, viabilizar el egreso de estudiantes en nueve meses, para hacer el programa competitivo y viable, 4 . La productividad del programa, hasta la fecha se han dictado cuatro cohortes, han egresado 32 alumnos y, están en proceso de tramitación del grado 12 alumnos, que completaran 44 egresados en cuatro años y, 5. El PEGU imparte un conocimiento necesario, es pertinente y tiene un alto potencial para dictarse a nivel nacional. En este sentido, hay que destacar el nuevo convenio a firmarse con la Carrera de Arquitectura de la Universidad Nacional Experimental del Táchira, UNET.

En la Tabla 1 se propone equiparar la UC a 12 horas con lo cual el PEGU tendría un total de 288 y 24 UC. Mientras se aprueba esta propuesta las materias de 2 UC se gestionarán en principio a través de 24 horas presenciales y 8 no presenciales. Por otro lado y para superar el sindrome todo menos tesis (TMT) se plantea la realización del Trabajo Especial de Grado (TEG) en los Seminarios.

Las cinco (5) fortalezas o claves del Programa de Gestión Urbana se pueden sintetizar en lo siguiente:

\section{Tabla 1}

Propuesta de Gestión del conocimiento para EI Programa De Gestion Urbana (PEGU)

\begin{tabular}{|c|c|c|c|c|c|c|c|c|}
\hline 1er. Trimestre & H & UC & 28. Trimestre & H & UC & 3er. Trimestre & $H$ & ve \\
\hline Introducción & 12 & * & Gestión Ambiental & 24 & 2 & Electiva 1 & 24 & 2 \\
\hline $\begin{array}{l}\text { Marco Legal de la } \\
\text { Gestión Urbana }\end{array}$ & 24 & 2 & Políticas Públicas & 24 & 2 & Electiva 2 & 24 & 2 \\
\hline $\begin{array}{l}\text { Planificación y } \\
\text { Gestión Estratégica } \\
\text { de Ciudades }\end{array}$ & 24 & 2 & $\begin{array}{l}\text { Formación Gerencial } \\
\text { y Liderazgo }\end{array}$ & 24 & 2 & Electiva 3 & 24 & 2 \\
\hline Seminario TEG 1 & 24 & 2 & Seminario TEG 2 & 36 & 3 & Seminario TEG 3 & 36 & 3 \\
\hline Total & 74 & 6 & & 96 & 9 & & 96 & 9 \\
\hline
\end{tabular}

Fuente: Comité Académico PEGU, elaboración propia 1999. 
- Una alianza estratégica que se fortalece, LUZ-PDVSA-ALCALDIAS del ZULIA: EI PEGU se formula en respuesta a la solicitud de LAGOVEN, hoy Petroléos de Venezuela Sociedad Anonima, PDVSA. Esta alianza ha financiado tres de las cuatro cohortes que se han dictado del programa $y$, ha creado una plataforma interinstitucional que viabilizó su acreditación por el CNU en 1998. El PEGU es el primer Programa de postgrado acreditado de la FADLUZ y esta dirigido a la formación, actualización y capacitación del personal profesional de las Alcaldias, Instituciones públicas y privadas $y$, profesionales vinculados con hacer ciudad y su objetivo es mejorar la gestión local y por ende la calidad de vida en las ciudades.

- Un programa transcisciplinario e Interinstitucional: El programa se diseñó considerando los objetivos y necesidades de educación y adiestramiento de profesionales vinculados a la gestión urbana, disciplina que por su naturaleza es transdisciplinaria e interinstiticional $y$, en consecuencia las materias obligatorias $y$ electivas que se dictan y, los profesores que participan responden a estos requerimientos.

- Una experiencia de Investigación+Docencia+Extensión=Academia $(I+D+E=A)$ : La primera cohorte del Programa, que egresó en Junio de 1996, se ha organizado en la Asociación Civil de Especialistas en Gestión Urbana, ACIVEGESUR y, ha participado como consultora conjuntamente con la Facultad de Arqui- tectura, FADLUZ, en la elaboración de siete proyectos de Gestión Urbana para las ciudades de la Costa Oriental del Lago de Maracaibo a través del Convenio LUZ- LAGOVEN-ALCALDÍAS DE LA COLM-ACIVEGESUR (1996-1997 y 1998-1999). Este planteamiento responde a la necesidad creciente de investigación aplicada que demanda el diseño de fórmulas de gestión nuevas dirigidas a promover y viabilizar el reconocimiento de estos proyectos como el vehículo por excelencia para integrar la docencia (pre y postgrado) - investigación y extensión con el medio y, solucionar problemas del entorno a corto plazo. La investigación aplicada al responder a las necesidades del medio incluye, como aspectos igualmente importantes y prioritarios, cumplir con la Visión de la Universidad, contextualizar (compromiso social y pertinencia) la investigación universitaria y la actualización docente a partir de la vinculación de la academia con la práctica y por ende mejorar la calidad de la docencia. Esta política se apoya en la consideración de tres aspectos clave: ranqueo, financiamiento e impacto. a. Ranqueo: la necesidad de LUZ y de la Facultad de Arquitectura de mejorar su productividad cognitiva, referida a formas más eficientes de producción y transferencia de conocimiento al sector productivo (García, 1996), para competir con éxito y lograr mejor presupuesto y ranqueo; $b$. Financiamiento: considera las dificultades de financiamiento por las que atraviesa recurrentemente LUZ y 
el financiamiento obtenido a través del Convenio, LUZ-PDVSA-ALCALDIAS DE LA COLM -ACIVEGESUA de aproximadamente 170 millones de Bolivares; y d. Impacto de la investigación extensiva en el ritmo de aprendizaje (García, 1996), en la creación de nuevo conocimiento y praxis, el apoyo a la formación de IV y $\mathrm{V}$ nivel y, en el crecimiento económico y competitivo del pais.

- Una plataforma generadora de conocimiento innovador, nueva teoría y praxis. El reglamento de los Estudios para Graduados (LUZ, 1998a), establece que los TEG, Artículo 109, "deben ser el resultado de una actividad de adiestramiento o de investigación que demuestre el manejo instrumental de los conocimientos obtenidos por el aspirante en la respectiva área". En este sentido, en el PEGU los Trabajos Especiales de Grado (TEG), están dirigidos fundamentalmente a desarrollar capacidades en los alumnos para intervenir en el medio urbano $y$, diseñar y gestionar soluciones a problemas urbanos municipales y/o a construir oportunidades para mejorar la gestión local y la calidad de vida urbana. Estos problemas son multidimensionales, complejos y dinámicos. La lógica de los TEG puede resumirse en la secuencia, ESTADO ACTUAL + ACCIÓN (ES) = ESTADO DESEABLEY POSIBLE, donde el estado actual puede ser una situación problema, una oportunidad o el logro de la VISIÓN. Según Crozier, "la crisis es una potente señal que conviene descifrar para transformarla en oportunidad". Los estudiantes a través de los Seminarios se enfrentarán a procesos continuos de solución de problemas existentes o potenciales, a partir del aprendizaje desde la experimentación y, de redes de relaciones, donde articulan teoria y praxis. Se trata de innovar, identificando y resolviendo incluso problemas que aún no se han presentado. En este sentido, según Crozier (1996: 24) "para innovar en sistemas complejos, no vasta la invención técnica, se necesitan las interacciones constructivas entre los eslabones de las cadenas de relaciones humanas, desde el conocimiento cientifico al descubrimiento técnico, es la capacidad de cooperación y de trabajar juntos". La necesidad de redefinir la docencia de postgrado a partir de una visión pluralista y postburocrática, que permita su articulación con el contexto global, la coexistencia de diferentes paradigmas de investigación y de distintos modos de organización de las funciones académicas básicas, en un contexto de autonomia y compromiso social, implica horizontalizar y democratizar los postgrados y promover y dar cabida a nuevas formas de investigación "de agenda" más flexibles y abiertas al contexto, que viabilice la investigación interuniversitaria, interinstitucional y transdisciplinaria, comprometida con las necesidades cambiantes de la sociedad. Se entiende por gestión postburocratica la que viabiliza 
formas de organización horizontal, flexible y, participativa, no vertical ni jerárquica (Cunill, 1997).

- Los TEG asumidos como Investigación de Agenda: las Agendas, son una manera de organizar y procesar las preguntas que se hacen los distintos actores, desde la perspectiva de sus propios intereses, complementando de esta manera las que se hacen los científicos. La Agenda Ciudad "es el escenario para la innovación donde se pretende articular la generación de conocimiento científico tecnológico con una diversidad de actores, sus problemas, capacidades $y$ recursos, para generar redes que faciliten la interacción cooperativa, la negociación y los esfuerzos para superar la crítica situación que caracteriza las ciudades" (CONICIT, 1997:3). Al asumir los TEG como investigaciones de agenda, que en el caso del PEGU tienen por objeto dar solución a los principales problemas urbanos y generar teoría en ese campo, "hará posible a mediano plazo catalogar las soluciones propuestas y las fórmulas de gestión generadas que puede derivar en un Reglamento de Gestión Urbana del que el pais carece" (Ferrer, 1998). En la Tabla 2 se contras-

Tabla 2

Gestión de los postgrados. Dos enfoques en tensión en LUZ

\begin{tabular}{|c|c|c|}
\hline & $\begin{array}{c}\text { Escenario Funcional } \\
\text { Plan Estratégico de LUZ y el REPG }\end{array}$ & $\begin{array}{c}\text { Escenario Emergente } \\
\text { ELPEGU }\end{array}$ \\
\hline Paradigma & $\begin{array}{l}\text { Funcional, burocrático, (gestión de la } \\
\text { estabilidad) }\end{array}$ & $\begin{array}{l}\text { Postburocratico, (gestión de la } \\
\text { incertidumbre) }\end{array}$ \\
\hline $\begin{array}{l}\text { Actoresi } \\
\text { decisiones }\end{array}$ & Lógica jerárquica, vertical y en cascada & $\begin{array}{l}\text { Hedes de cooperación } \\
\text { Actores polivalentes que comparten una } \\
\text { visión }\end{array}$ \\
\hline Escenario & Cerrado, autoreferencial & Abierto al contexto $y$ al benchmarking \\
\hline $\begin{array}{l}\text { Docencia de } \\
\text { Postgrado }\end{array}$ & $\begin{array}{l}\text { Función, eje de la transformación } \\
\text { académica de LUZ }\end{array}$ & $\begin{array}{l}\text { gestión del conocimiento, } \\
\text { transdisciplinar, interinstitucional, } \\
\text { alianzas estratéglcas, redes }\end{array}$ \\
\hline Visión & Fragmentada por funciones & Global e integradora \\
\hline Universidad & $\begin{array}{l}\text { De la excelencia con compromiso } \\
\text { social, acuerdo a través de } \\
\text { concesiones }\end{array}$ & $\begin{array}{l}\text { De calidad y rentable socialmente = } \\
\text { produce beneficios a la sociedad, } \\
\text { genera impacto evaluable y } \\
\text { reconocible }\end{array}$ \\
\hline Academia & Tres funciones básicas separadas & $\begin{array}{l}1+D+E=\text { Academia, actividades } \\
\text { inseparables }\end{array}$ \\
\hline Modelo & Prescriptivo del "debe ser" & Posracional, inductivo y critico \\
\hline Posicion & Cultura de la inconsecuencia, todo vale & $\begin{array}{l}\text { Cultura del desempeño, premia los } \\
\text { logros }\end{array}$ \\
\hline
\end{tabular}

Fuente: Ferrer, M., elaboración propia 1999. 
tan, a partir del análisis de nueve variables seleccionadas, los dos escenarios descritos anteriormente.

\section{Tres reflexiones finales}

\subsection{Reformulación del Programa de Especialización en Gestión Urbana}

La propuesta de cambio del PEGU y, la nueva concepción de los $T E G$, se fundamenta en el aprendizaje obtenido en la gestión de la primera cohorte del programa, a partir de la cual se pueden señalar tres conclusiones básicas: a. la metódica utilizada, que igualó los requerimientos de los TEG de los Programas de Especialización, a la formulación de anteproyectos de investigación, siguiendo las pautas del reglamento de EPG, las "Normas Mínimas para presentar Trabajos Escritos en LUZ" y del asesor metodológico, estandarizó su alcance y resultados, desaprovechando el potencial de creación de nuevo conocimiento y praxis de 32 alumnos. b. Paralelamente permitió identificar las múltiples vertientes y preocupaciones o valoraciones colectivas de los funcionarios municipales sobre la construcción y mantenimiento de la ciudad y del municipio, desde una perspectiva interinstitucional y transdisciplinar, en respuesta a una realidad urbana que es compleja, dinámica, fragmentada y que intenta posicionarse en el mercado de las ciudades y, c. reconoce el alto potencial tanto del PEGU, para conformarse como plataforma generadora de nuevo conocimiento como de los TEG, para calificar como proyectos de Agenda.
A partir de las consideraciones anteriores la nueva propuesta del PEGU implica cuatro cambios fundamentales en el Reglamento de Estudios para Graduados: 1. Homologar la Unidad Crédito a estándares latinoamericanos (UE $=12$ horas) 2. Exigir un máximo de 24 UC para las Especializaciones incluyendo los TEG y, tres trimestres, de 12 semanas $\mathrm{c} / \mathrm{u}$ de escolaridad. 3. Bajar el tiempo de permanencia en la Especialización a dos años y, 4. Crear un Programa de Investigación de Agenda en Gestión Urbana desde la DEPG que se articule, a través de líneas de investigación compartidas (interfacultades e interuniversidades, nacionales y extranjeras), en redes virtuales de los postgrados vinculados a la temática urbana-local.

Para viabilizar las propuestas, se plantean cinco estrategias que se formularon considerando la realidad actual que exige, flexibilidad, flujos de información más libres, respuestas instantáneas $e$ imaginativas y eliminar tramites para conseguir el efecto deseado:

- Asumir la gestión y el marketing del capital intelectual de la universidad como idea fuerza para REDEFINIR y FLEXIBILIZAR los estudios de postgrado y adecuarlos a las demandas del contexto.

- Cambiar la visión funcional y la práctica burocrática en los EPG para promover postgrados abiertos y flexibles, en red y homologados internacionatmente con el apoyo y potencial que significa el caudal de convenios firmados por LUZ y la educación digital.

- Asumir la investigación de agenda, dirigida a aprender a diseñar y gestionar soluciones, aprender a empren- 
der, innovar, tramitar y gerenciar problemas, oportunidades y/o el logro de la VISIÓN, en un contexto postburocrático, abierto y plural, que promueva la divergencia.

- Potenciar el aprendizaje, a partir de la creación de redes virtuales de docencia e investigación, con universidades del país y extranjeras $y$, del benchmarking, o mejores prácticas de postgrado.

- Diseñar una política de mercadeo y divulgación de los programas de postgrado que asegure su competitividad y rentabilidad.

\subsection{Rearquitectura de la universidad y de los estudios de postgrado}

Existe una realidad local y global, que nadie puede desconocer ni subestimar y que impone la necesidad de nuevos enfoques y actitudes mentales ya que, si nos apartarnos de esa realidad, corremos el riesgo de ser irrelevantes. Según Toffler (2000), "en plena REVOLUCIÓN DIGITAL, el conocimiento es en si mismo, la principal forma del capital accesible a todos y, si lo utilizamos de forma creativa puede realmente generar más conocimiento. Por otro lado, "el conocimiento que no es un recurso escaso, está generando una nueva economía que es verdaderamente revolucionaria". En este contexto y, según Toffler, América Latina tiene que correr con mayor velocidad para alcanzar las economias más avanzadas y, ya comienza a hacerlo. Para lograrlo, es necesario asumir la educación de postgrado como "el proceso mediante el cual las personas adquieren la capaci- dad de redefinir constantemente la cualificación necesaria para realizar tareas determinadas y de acceder a las fuentes y métodos, para adquirir dicha cualificación" (Castells, 1998). Según este autor, quien posee educación puede autoprogramarse, trabajador autoprogramabley, por el contrario el trabajador genérico, no educado sólo calificado, no tiene esa capacidad y se hace prescindible.

La rearquitectura o nueva arquitectura del capital intelectual (Cl), implica el desarrollo y aplicación de nuevas herramientas tecnológicas, educación digital, para acelerar la creación de conocimiento innovador $(l+D)$, el proceso de aprendizaje, la distribución y acceso democrático a ese conocimiento y, la promoción de actitudes emprendedoras. En este contexto, la universidad tiene el potencial para convertirse en la empresa red emergente y estratégica, que viabilizará, la asunción de nuevos paradigmas en un clima organizacional democrático, participativo, plural y, promotor de innovación y creatividad, requisito básico para lograr el crecimiento sostenido de Venezuela. La universidad así concebida, es el BETA SITE o LABORATORIO DE REINVENCIÓN E innovación que, según Osborne y Plastrik (1998), "es donde se aplican y, generan las nuevas ideas o trabajo pionero, crea sus propias reglas, realiza una gestión directa $y$, se protege contra intromisiones". Es una incubadora de ideas, proyectos y conocimiento innovador (investigación en extensión), promueve el desarrollo personal de la comunidad universitaria y desarrolla procesos que implican aprender a asociarnos, a crear y a emprender. 
Los principios clave para lograr el cambio son cuatro: asumir la Investigación, Docencia y Extensión = Academia, como actividades inseparables en el hacer universitario, ser actores polivalentes, generadores de rentabilidad social que comparten la VISIÓN, en un contexto que suma voluntades (ganar -ganar), igualdad de oportunidades, para generar un ambiente de confianza activa., "la equidad tiene efectos positivos sobre el desarrollo del potencial asociativo y la innovación" y, alianzas para construir redes que promuevan la creación de conocimiento y praxis innovadoras. Las estrategias para la rearquitectura de la universidad y los postgrados son dos: construir sobre to construido, apoyando proyectos e iniciativas exitosas y a los emprededores e innovadores, escenario emergente $y$, construir oportunidades para permitir la participación activa y generar el cambio, utilizando el benchmarking como instrumento. Ahora ¿Cómo convertirnos en emprendedores? Asumiendo el paso de un mundo de la obediencia a un mundo de responsabilidad, que se apoya en la inteligencia creativa de los distintos actores (expertos en sus procesos), para conformar redes interactivas que permiten innovar $y$ aprender mediante el intercambio y, la cooperación para hacer frente mejor a los retos del mundo contemporáneo.

¿Cómo gestionar el cambio? A través de una dirección estratégica proactiva que suma voluntades $y$ liderazgos $y$ viabiliza, la pluralidad y el pensamiento divergente, la coexistencia de actores con posturas divergentes, democracia participativa, que comparten la visión y asumen el cambio como reto. La descentralización y flexibilidad organizativa, como medios para manejar la incertidumbre $y$, un entorno complejo, que demanda la evaluación continua de las necesidades de la sociedad y de las soluciones propuestas, es decir, investigación extensiva o aplicada permanente y, la organización en redes, en las que se desarrollan actitudes de cooperación, coordinación y promoción, características clave de los equipos de trabajo virtuales del mundo contemporáneo. En este sentido y, para lograr la transformación de la universidad es necesario generar un pacto a partir de una nueva cultura de gestión del capital intelectual universitario, entre la universidad y el país, que incluya a las organizaciones empresariales, el tercer y cuarto sector, los gremios y los sindicatos para salir del atraso y, en el marco de ese pacto elaborar un PLAN DE FORMACIÓN PROFESIONAL donde los postgrados tienen un rol protagónico (Castells, 1999).

Esta propuesta integra el conocimiento basado en el sentido común, el conocimiento basado en la técnica y, el conocimiento derivado de intereses sociales, en un proceso secuencial que respeta la especificidad de cada uno de ellos $y$, los aportes que pueden hacerse cuando se proponen y gestionan soluciones, en situaciones donde convergen múltiples actores, valores e intereses (Cunill, 1997). Por otro lado, esta propuesta implica una intervención directa moderna, entendida según Crozier (1996:87) "como conjunto de acciones convergentes, coordinadas, pero en absoluto coherentes en el sentido jerárquico del térmi- 
Los principios clave para lograr el cambio son cuatro: asumir la Investigación, Docencia y Extensión = Academia, como actividades inseparables en el hacer universitario, ser actores polivalentes, generadores de rentabilidad social que comparten la VISIÓN, en un contexto que suma voluntades (ganar -ganar), igualdad de oportunidades, para generar un ambiente de confianza activa., "la equidad tiene efectos positivos sobre el desarrollo del potencial asociativo y la innovación" y, alianzas para construir redes que promuevan la creación de conocimiento y praxis innovadoras. Las estrategias para la rearquitectura de la universidad y los postgrados son dos: construir sobre lo construido, apoyando proyectos e iniciativas exitosas y a los emprededores e innovadores, escenario emergente $y$, construir oportunidades para permitir la participación activa y generar el cambio, utilizando el benchmarking como instrumento. Ahora ¿Cómo convertirnos en emprendedores? Asumiendo el paso de un mundo de la obediencia a un mundo de responsabilidad, que se apoya en la inteligencia creativa de los distintos actores (expertos en sus procesos), para conformar redes interactivas que permiten innovar y aprender mediante el intercambio y, la cooperación para hacer frente mejor a los retos del mundo contemporáneo.

¿Cómo gestionar el cambio? A través de una dirección estratégica proactiva que suma voluntades y liderazgos y viabiliza, la pluralidad y el pensamiento divergente, la coexistencia de actores con posturas divergentes, democracia participativa, que comparten la visión y asumen el cambio como reto. La descentralización y flexibilidad organizativa, como medios para manejar la incertidumbre $y$, un entorno complejo, que demanda la evaluación continua de las necesidades de la sociedad y de las soluciones propuestas, es decir, investigación extensiva o aplicada permanente $y$, la organización en redes, en las que se desarrollan actitudes de cooperación, coordinación y promoción, características clave de los equipos de trabajo virtuales del mundo contemporáneo. En este sentido $y$, para lograr la transformación de la universidad es necesario generar un pacto a partir de una nueva cultura de gestión del capital intelectual universitario, entre la universidad y el pais, que incluya a las organizaciones empresariales, el tercer y cuarto sector, los gremios y los sindicatos para salir del atraso y, en el marco de ese pacto elaborar un PLAN DE FORMACIÓN PROFESIONAL donde los postgrados tienen un rol protagónico (Castells, 1999).

Esta propuesta integra el conocimiento basado en el sentido común, el conocimiento basado en la técnica $y$, el conocimiento derivado de intereses sociales, en un proceso secuencial que respeta la especificidad de cada uno de ellos $y$, los aportes que pueden hacerse cuando se proponen y gestionan soluciones, en situaciones donde convergen múltiples actores, valores e intereses (Cunill, 1997). Por otro lado, esta propuesta implica una intervención directa moderna, entendida según Crozier (1996:87) "como conjunto de acciones convergentes, coordinadas, pero en absoluto coherentes en el sentido jerárquico del térmi- 
egresados de pre y postgrado, investigación y extensión de calidad, que soluciona problemas y construye oportunidades.

La universidad del siglo XXI, será capaz de construir en un entorno en constante transformación e incertidumbre, conocimiento y praxis innovadoras $y$, analizar e intervenir, en los aspectos clave de la realidad local y global a partir de estilos de gestión plural donde, la investigación, docen* cia y extensión son actividades académicas inseparables $y$, el personal que trabaja en redes es polivalente.

Esta universidad, abierta y plural, no funcionalista, posburocrática, crítica, menos modelizable e inductiva, produce beneficios (económicos y sociales) $y$, agrega valor, es decir participa en el crecimiento rentable y sostenido del pais. La UNIVERSIDAD que asume la educación, innovación y creación de conocimiento aplicable como recurso inagotable, impulsor de la economía y creador de riqueza (Toffler, 1997) y, lo gestiona y mercadea potenciando su $\mathrm{Cl}$, cumple con su misión y logra su visión y en consecuencia, FORMA PARTE DEL SECTOR PRODUCTIVO DEL PAÍS. Esto sólo será posible cuando de la universidad homologada de la cultura de la inconsecuencia o todo vale, pasemos a la universidad heterologada de la cultura del reconocimiento por desempeño donde, los resultados cuentan (Bardwick en Pinedo, 1998).

La universidad rentable socialmente promueve el potencial de la sociedad para organizarse (fortalecimiento de la sociedad civil o tercer sector según Cunill), en un contexto de confianza y partici- pación activa, donde confluyen actores que quiebran las pautas tradicionales y las divisiones funcionales y tecnológicas. Un estilo relacional de gobierno, según Goma y Brugué (1998), "implica gobernar a través de redes de actores interdependientes $y$, tiene que ver más, con la influencia que con el ejercicio de autoridad de jerarquias decisionales legalmente establecidas". En ese sentido Crozier (1996: 66 y 52) plantea que "se debe invertir masivamente en el desarrollo del conocimiento de los sistemas humanos reales y, en la formación de los responsables del razonamiento moderno, que sobrepase la lógica vertical del ordenamiento llamado democrático, pero que de hecho es burocrático" $y$, en consecuencia, "debemos pasar de la democracia de acceso a la democracia de deliberación".

En definitiva, la lógica de lo relacional - comunicacional, en un marco más pluralista de actores, intereses e, interacciones, potencia el $\mathrm{Cl}$ universitario $\mathrm{y}$ los estudios de postgrado como plataforma generadora de conocimiento innovador que, en este contexto, es la actividad emergente y la estrategia de la universidad con rentabilidad social que agrega valor $y$, viabiliza, el crecimiento sostenido del país.

\section{Bibliografía citada}

Blair, Tony (1998). La Tercera Via. Grupo Santillana de Ediciones SA. Madrid.

Bresser Pereira, Luiz Carlos (1998). EI Estado del Siglo XXI: tendencias, desafios y capacidades necesarias. Conterencia dictada en el lil Congreso internacional del CLAD. Madrid. 
Browning, John y Reiss, Spencer (2000). El crecimiento es la clave de la nueva economia. En, El Universal, página 2-12, del 4 de Junio.

Byron, Christopher (2000). El lado oscuro En Revista, Business Technology, Mayo.

Castells, Manuel (1998). La era de la información: economia, sociedad y cultura. Vol. 3. Fin de milenio. Alianza Editorial. Madrid.

Castells, Manuel (1998a). "Hacia el Estado Red". Ponencia presentada en Seminario Sociedad y Reforma del Estado, Ministerio de Administración Federal de Brasil.

Castells, Manuel (1999). La transformación del Trabajo. La Factoria Cultural. Barcelona.

Crozier, Michel (1996). La crisis de la inteligencia, ensayo sobre la incapacidad de las élites para reformarse. Publicaclón del MAP. Madrid. España.

CONICIT (1997). Agenda Ciudad. Informe preliminar I Taller, Gerencia de Investigación Orientada. Caracas.

Cunill, Nuria (1997). Repensando lo Público a través de la Sociedad. CLAD. Editorial Nueva Sociedad. Caracas.

Frances, Antonio (1998). "El juego de los escenarios". En, Debates IESA, Vol. 4, No. 2. Caracas.

Ferrer, Mercedes (1997). Los postgrados en un contexto global. Una visión prospectiva, Ponencia presentada en las IV Jornadas de postgrado Vicerrectorado Académico. LUZ.

Ferrer, Mercedes (1998). Justo a tiempo el PEGU entro en Agenda. Ponencia presentada en el ler. Seminario de Investigación IFA-FADLUZ.

Ferrer, Mercedes (1998a). Investigación en Extensión. Ponencia presentada en el $\checkmark$ Seminario de Investigación CONDES, Vicerrectorado Académico. LUZ.
Ferrer, Mercedes (1999). Gestion y Marketing del conocimiento en Postgrado. La Especializacion en GestionUrbana apuesta por el cambio. Ponencia presentada en el Congreso Nacional de Educacion Avanzada, Vicerrectorado Académico. LUZ. Maracaibo.

Fukuyama, Francis (1994). El Fin de la Historia y el último Hombre. Planeta Colombia SA.

Fukuyama, Francis (1996). CONFIANZA, (Trust). 2a. Edición, Editorial Atlántida. Argentina.

Garcia, Carmen (1996). Conocimiento, Educacion Superior y Sociedad en Americalatina. Editorial Nueva Sociedad. Caracas.

Gates, Bill (1999). Los negocios en la era digital. Plaza \& Janés S.A.

Guiddens, Anthony (1996). Mas alla de la izquierda y la derecha. El futuro de las politucas radicales. Ediciones Catedra SA. Madrid.

Guedez, Victor (1998). Gerencia, cultural y educación. Fondo Editorial Tropykos. CLACDEC.Caracas.

Goma y Brugué (1998). Gobiernos locales y políticas públicas. Ariel Ciencia Politica. Madrid.

Huntington, Samuel (1997). El choque de las civilizaciones y la reconfiguracion del orden mundial. Editorial Paidos. Barcelona.

Kennedy, Paul (1993). Hacia el siglo XXI. Plaza \& Janes. Barcelona.

La Universidad del Zulia (1998). Plan de Desarrollo Estratégico. Astro Data. Maracaibo.

La Universidad del Zulia (1998a). Reglamentos de Estudios para Graduados. Ga* ceta Universitaria. Edición Extraordinaria, Reglamentos - Volumen XI. Maracaibo. 
Lewis, Michael (2000). Boom o Ficción. En Business Technology, Mayo. Venezuela.

Osborne, David y Plastrik, Peter (1998). La Reducción de la burocracia, cinco es: trategias para reinventar el gobierno. Editorial Paidós. Barcelona.

Samuelson, Paul (2000). Es preciso controlar la burbuja de Wall Street. En El Universal, pagina 2-2 del 28 de Mayo.

Pinedo, Diana (1998). Optimizar el riesgo: clave de los Buenos Negocios. Reseña del libro en, Debates IESA, Vol. 4 , No. 2 .
Toffler, Alvin (1997). El Cambio de poder. Editorial Plaza y Janes, 6a. edición. Barcelona.

Toffler, Alvin y Heidi (2000). El Estado de la revolución digital, momento titular para la historia. En El Universal, pagina 1-6, del 21 de Mayo.

Touraine, Alain (1999). ¿Cómo salir del liberalismo? Paidós, Estado y Sociedad. Barcelona.

Fernández, Froilan (2000). Red de Lenguajes. En EI Nacional, pagina $E / 6$, del 28 de Mayo. 